\title{
Melting of the moving vortex lattice in the presence of disorder*
}

\author{
A.E. Koshelev, ${ }^{1}$ and V.M. Vinokur \\ Materials Science Division \\ Argonne National Laboratory, Argonne, Illinois 60439
}

4th International Conference on Materials and Mechanisms of Superconductivity, High Temperature Superconductors (M²S-HTSC IV), Grenoble, France, July 5-9, 1994 (to be published in Physica C)

The submitted manuscript has ben authored by
a contractor $\alpha$ the U.S. Government under
contract No. W-31-109-ENG-38. Accordingly.
the U.S. Government retains a nonexclusive,
royalty-iree license to publish or reproduce the
published form of this contribution, or allow
others to do so, for U.S. Govemment purposes.

\section{DISCLAIMER}

This report was prepared as an account of work sponsored by an agency of the United States Government. Neither the United States Government nor any agency thereof, nor any of their employees, makes any warranty, express or implied, or assumes any legal liability or responsibility for the accuracy, completeness, or usefulness of any information, apparatus, product, or process disclosed, or represents that its use would not infringe privately owned rights. Reference herein to any specific commercial product, process, or service by trade name, trademark, manufacturer, or otherwise does not necessarily constitute or imply its endorsement, recommendation, or favoring by the United States Government or any agency thereof. The views and opinions of authors expressed herein do not necessarily state or reflect those of the United States Government or any agency thereof.

\footnotetext{
*Work supported by the U.S. Department of Energy, BES-Materials Sciences under contract \#W-31-109-ENG-38 and the National Science Foundation Office of Science and Technology Centers for Superconductivity under contract \#DMR91-20000.

lPermanent address: Institute of Solid State Physics, Chernogolovka, Moscow, Russia.
} 


\section{DISCLAIMER}

Portions of this document may be illegible in electronic image products. Images are produced from the best available original document. 


\title{
Melting of the moving vortex lattice in the presence of disorder
}

\author{
A.E.Koshelev * and V.M.Vinokur
}

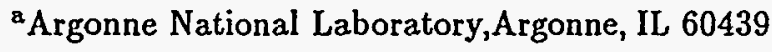

We investigate the melting of the two-dimensional vortex lattice moving in an inhomogeneous environment under the applied current $j$. We predict the existence of a dynamic phase transition at some current $j=j_{t}$ (crystallization current) from the motion of the amorphous configuration at $j<j_{t}$ to the motion of the crystal at $j>j_{i}$. $j_{t}$ exceeds essentially the critical current $j_{c}$ for strongly disordered systems and approaches $j_{c}$ with the decrease of the degree of disorder. We find that $j_{t}$ diverges as temperature approaches the melting temperature of the undisturbed lattice.

\section{Introduction}

The static and dynamic properties of the vortex state are strongly affected by the presence of disorder. Quenched disorder breaks down the crystalline order of the vortex lattice [1] and can convert the melting transition expected for vortex configuration in clean samples into a crossover between the liquid and amorphous states. The main effect of disorder on the vortex motion is onset of the critical current $j_{c}$ separating the pinned state at small driving currents, $j<j_{c}$, from the steady vortex motion at $j>j_{c}$. In this paper we predict new dynamic phenomenon: the dynamic phase transition between the coherent (at high currents) and incoherent (at small currents) regimes of vortex motion.

The effect of disorder on the vortex motion is usually described in terms of the averaged pinning force equivalent to an additional (nonlinear) friction. Here we address the effect of the fluctuating component of the pinning force on the melting transition of moving lattice. At large velocities the pinning force can be described as the additional stochastic force $F_{p}(r, t)$ and characterized by the effective "shaking" temperature $T_{s h}$. At $T<T_{m}$ the vortex lattice experiences a dynamic phase transition at a certain current $j_{t}(T)$ from the motion of a vortex crystal at $j>j_{t}$ to the motion of an amorphous vortex configuration at $j<j_{t}$. The transition (crystallization) current $j_{\imath}$ diverges near $T_{m}$.

The transition between different regimes of vor-

- Permanent address: Institute of Solid Slate Physics, Chernogolouka, Moscow, Russia tex motion was observed experimentally as a jump-like transition in Ref. [2] and as a continuous transition in Ref. [3].

\section{Analitical estimates}

We consider the $2 \mathrm{D}$ system of vortices subject to a disorder potential $U(r)$ generated by the randomly distributed pinning centers, $\left\langle U(\mathbf{r}) U\left(\mathbf{r}^{\prime}\right)\right\rangle=\gamma_{U} f\left(\mathbf{r}-\mathbf{r}^{\prime}\right)$, with $\int \mathrm{d} \mathbf{r} f(\mathbf{r})=1$. We assume $f(\mathbf{r})=\exp \left(-r^{2} / 2 r_{p}^{2}\right) /\left(2 \pi r_{p}^{2}\right)$. Consider the regime of fast motion of the vorices under the action of an external force $F_{\text {ext }}=(B / c) j$ much larger than the critical force $F_{c}$. To investigate the fluctuations of the vortex motion in the disordered media we find the correlation function $\aleph_{\alpha \alpha^{\prime}}(\mathbf{r}, t)=\left\langle F_{p \alpha}(0,0) F_{p \alpha^{\prime}}(\mathbf{r}, t)\right\rangle$ of the random forces $\mathrm{F}_{p}=-n(\mathrm{r}, t) \nabla U(\mathrm{r}-\mathrm{v} t)$, acting on the moving vortex liquid within the first order perturbation theory:

$\aleph_{\alpha \alpha^{\prime}}(\mathbf{r}, t)=n_{v} \gamma_{U} \delta(\mathbf{r}) \nabla_{\alpha} \nabla_{\alpha^{\prime}} f(\mathbf{v} t)$.

Here $n(r, t)$ is the vortex density, $n_{v}=\langle n(r, t)\rangle$, $v=F_{\text {ext }} / \eta$ is the lattice velocity, and $\eta$ is the viscosity friction coefficient. $\aleph_{\alpha \alpha^{\prime}}$ resembles the correlation function for the Langevin force up to anisotropy with respect to indices $\alpha$ and $\alpha^{\prime}$. This does not influence much on the response of the vortex system and the displacements produced by $F_{p}$ are similar to thermal ones. Therefore, it is natural to define the effective "shaking" temperature characterising the amplitude of $F_{p}$ :

$T_{s h}=\frac{1}{4 \sqrt{2 \pi}} \frac{n_{v} \gamma U}{F_{e x t} r_{r}^{3}}$. 
The effective temperature of the vortex system is then $T_{\text {eff }}=T+T_{s h}$. The estimate for the "crystallization" force, $F_{t}$, can be obtained from the condition $T_{\text {eff }}\left(F_{\text {ext }}=F_{t}\right)=T_{m}$, where $T_{m}$ is the melting temperature of the vortex lattice. This gives:

$$
F_{t}=\frac{n_{v} \gamma_{U}}{4 \sqrt{2 \pi}\left(T_{m}-T\right) r_{p}^{3}}
$$

This estimate is valid when $F_{z} \gg F_{c}$. For $T=0$. this corresponds to the case of strong disorder $\gamma_{U}^{1 / 2} / r_{p}>T_{m}$. In the case of weak disorder the dynamic transition at $T=0$ occurs slightly above $F_{c}$. However in the vicinity of $T_{m}$ the force $F_{c}$ drops due to the thermal smearing of the random potential while the force $F_{t}$ diverges.

\section{Numerical simulations}

To verify the above predictions we performed simulations of the motion of point vortices by numerical solution of the Langevin equations for the vortex coordinates $\mathbf{R}_{\boldsymbol{i}}$ :

$\frac{\mathrm{d} \mathbf{R}_{i}}{\mathrm{~d} t}=\mathbf{f}_{i}^{v-v}-\nabla U\left(\mathbf{R}_{i}\right)+\mathbf{f}_{T i}(t)+\mathbf{f}_{e x t}$.

Here $f_{i}^{v-v}$ is the force acting on $i$-th vortex from the other vortices, $U(\mathbf{r})$ is the random potential, $\mathbf{f}_{e x t}$ is the external force, and $\mathbf{f}_{T i}(t)$ is the Langevin force. The quantity $s \Phi_{0}^{2} /(4 \pi \lambda)^{2}$ and the lattice spacing $a_{0}$ of the ideal lattice are taken as units of energy and length. Simulations have been performed for two sets of parameters corresponding to a "dirty" system with individual pinning $\left(\sqrt{\gamma U} /\left(T_{m} r_{p}\right) \approx 2.9\right)$ and to a cleaner system at the "plastic-elastic" crossover $\left(\sqrt{\gamma_{U}} /\left(T_{m} r_{p}\right) \approx 2.0\right)$.

We solved the equations (4) at different values of the $\mathrm{f}_{\text {ext }}$ and $T$. At each choice of $\mathrm{f}_{\text {ext }}$ and $T$ we calculated the concentration of lattice defects using the triangulation procedure [4]. We started from high forces where the disorder in the lattice is small. At any temperature below $T_{m}$ there is a well defined force $f_{t}(T)$ at which the concentration of defects sharply increases. The numerically obtained phase diagram "temperature-force" is shown in Fig. 1 . We found $f_{t}(0) / f_{c}(0) \approx 2.4$ and $\approx 1.5$ for the "dirty" and "clean" system correspondingly. At low temperatures the transition is accompanied by the increase of the dynamic friction force. This effect is more pronounced for the "clean" system and is strongly suppressed by the thermal fluctuations.

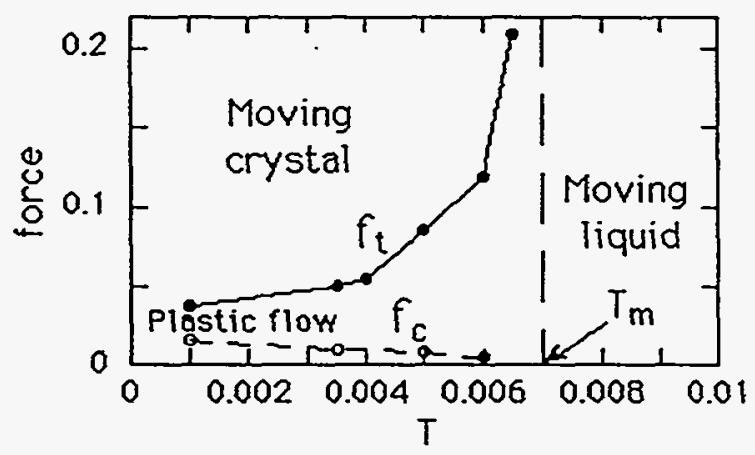

Figure 1. The phase diagram for the "dirty" system. The line $f_{t}(T)$ corresponds to the dynamic phase transition. $f_{c}(T)$ is the critical current corresponding to the crossover between flux flow and thermally assisted creep.

\section{Acknowledgments}

Simulations have been performed on the SUN Workstations in the Kamerlingh Onnes Laboratory (Leiden, The Netherlands) and in the Mathematics and Computer Science Division of Argonne National Laboratory. This work was supported by the National Science Foundation Office of the Science and Technology Center under contract No. DMR 91-20000 and by the U.S. Department of Energy, BES-Materials Sciences, under contract No. W-31-109-ENG-38.

\section{REFERENCES}

1. A.I.Larkin, Zh. Eksp. Teor. Fiz. 58, 1466 (1970)

2. R.Wördenweber and P.H.Kes, Phys.Rev., B 34, 494 (1986).

3. S.Bhattacharya and M.J.Higgins, Phys. Rer. Lett. 70, 2617 (1993)

4. F.F.Preparata, M.L.Shamos. Computational Geometry: An Intraduction. Springer-Verlag. New York, 1985. 